\title{
Article \\ Asphalt Mixture with Scrap Tire Rubber and Nylon Fiber from Waste Tires: Laboratory Performance and Preliminary M-E Design Analysis
}

\author{
Dongzhao Jin (D), Dongdong Ge ${ }^{(D)}$, Xiaodong Zhou and Zhanping You *
}

check for updates

Citation: Jin, D.; Ge, D.; Zhou, X.; You, Z. Asphalt Mixture with Scrap Tire Rubber and Nylon Fiber from Waste Tires: Laboratory Performance and Preliminary M-E Design Analysis. Buildings 2022, 12, 160. https://doi.org/10.3390/ buildings 12020160

Academic Editors: Ahmed B. Senouci and Walid Maherzi

Received: 22 December 2021

Accepted: 27 January 2022

Published: 2 February 2022

Publisher's Note: MDPI stays neutral with regard to jurisdictional claims in published maps and institutional affiliations.

Copyright: (C) 2022 by the authors. Licensee MDPI, Basel, Switzerland. This article is an open access article distributed under the terms and conditions of the Creative Commons Attribution (CC BY) license (https:// creativecommons.org/licenses/by/ $4.0 /)$.
Department of Civil and Environmental Engineering, Michigan Technological University, 1400 Townsend Drive, Houghton, MI 49931-1295, USA; dongj@mtu.edu (D.J.); dge1@mtu.edu (D.G.); xzhou3@mtu.edu (X.Z.)

* Correspondence: zyou@mtu.edu

\begin{abstract}
Scrap tire rubber and nylon fiber are waste materials that could potentially be recycled and used to improve the mechanical properties of asphalt pavement. The objective of this research was to investigate the properties of scrap tire rubber and nylon fiber (R-F) modified warm mix asphalt mixture (WMA). The high-temperature performance was estimated by the Hamburg wheel-tracking testing (HWTT) device. The low-temperature cracking performance was evaluated by the diskshaped compact tension (DCT) test and the indirect tensile strength (IDT) test. The stress and strain relationship was assessed by the dynamic modulus test at various temperatures and frequencies. The extracted asphalt binder was evaluated by the dynamic shear rheometer (DSR). Pavement distresses were predicted by pavement mechanistic-empirical (M-E) analysis. The test results showed that: (1) The R-F modified WMA had better high-temperature rutting performance. The dynamic modulus of conventional hot mix asphalt mixture (HMA) was 21.8\% 103\% lower than R-F modified WMA at high temperatures. The wheel passes and stripping point of R-F modified WMA were 2.17 and 5.8 times higher than those of conventional HMA, respectively. Moreover, the R-F modified warm mix asphalt had a higher rutting index than the original asphalt. (2) R-F modified WMA had better cracking resistance at a low temperature. The failure energy of the R-F modified WMA was $24.3 \%$ higher than the conventional HMA, and the fracture energy of the R-F modified WMA was 7.7\% higher than the conventional HMA. (3) The pavement distress prediction results showed the same trend compared with the laboratory testing performance in that the R-F modified WMA helped to improve the IRI, AC cracking, and rutting performance compared with the conventional HMA. In summary, R-F modified WMA can be applied in pavement construction.
\end{abstract}

Keywords: scrap tire rubber and nylon fiber; dynamic modulus; dynamic shear modulus; Hamburg wheel-track testing; disk-shaped compact tension; indirect tensile strength; pavement M-E

\section{Introduction}

Scrap tire rubber and nylon fiber are waste materials from disposal tires, and they may cause negative effects on the environment. Millions of tires are generated every year due to a growing number of vehicles [1,2]. Nylon fiber and scrap tire rubber are two components of scrap tires [3]. Recycling used tire rubber and nylon fiber in asphalt pavement is one of the most effective methods to dispose of tire rubber and nylon fiber to prevent an increasing environmental hazard [4-6].

Researchers have focused on nylon fiber modified asphalt mixture performance. Yin et al. [7] found that nylon fiber could increase high-temperature deformation resistance and low-temperature cracking resistance for stone matrix asphalt mixture. Lee et al. [8] stated that asphalt mixture modified by fiber particle shows improved fatigue cracking resistance compared with conventional asphalt mixture. Taherkhani et al. [9] found that nylon fiber modified asphalt mixture is more resistant to high-temperature deformation and fatigue cracking than asphalt mixture with added nano-clay modifier. Meanwhile, 
the mixing temperature reduction technology of asphalt as an alternative technique has also been attracting more and more attention. Yang et al. [10] studied the characterization of scrap rubber modified warm mix asphalt pavement and found that the warm mix of asphalt helped to reduce the consumption of asphalt and fuel and the release of hazardous emissions. Additionally, the warm mix of asphalt mixture has comparable resistance to high-temperature deformation and low-temperature cracking and better fatigue properties compared with scrap rubber hot mix asphalt mixture. Oliveira et al. [11] reported that the warm mix additive in asphalt mixture could help reduce the temperature by $30{ }^{\circ} \mathrm{C}$ to complete the mix and compaction. Dinis-Almeida et al. [12] also mentioned that warm mix technology helps to reduce the emission of $\mathrm{CO}_{2}$.

Scrap rubber as an additive in asphalt mixture also has numerous benefits, and many researchers have studied scrap rubber incorporated with asphalt mixture by wet process, dry mix, or terminal blend. Airey et al. [13] found that one component from asphalt was consumed by scrap rubber particle, and it caused the scrap rubber to swell after contact with the asphalt binder. Rahman et al. [14] studied scrap rubber incorporated with asphalt mixture by the dry blend method and concluded that it is more sensitive to moisture susceptibility compared with hot asphalt mixture. Chen et al. [15] found that ground tire rubber modified gap graded asphalt mixture prepared by the dry process has a higher resistance to low-temperature cracking and high-temperature rutting compared with control asphalt mixture. Xie et al. [16] reported that the scrap rubber modified asphalt mixture prepared by terminal blend has better pavement performance than by the dry or wet process.

A thorough literature review found that both nylon fiber and rubber could help to improve asphalt mixture performance. However, there is little research on both nylon fiber and rubber and on Sasobit as an additive in asphalt mixture to produce scrap tire rubber and nylon fiber modified WMA. Therefore, the main objective of this research was to investigate the properties of scrap tire rubber and nylon fiber modified warm asphalt mixture. The high-temperature properties were estimated by Hamburg wheel-track testing (HWTT). The low-temperature cracking performance was evaluated by the disk-shape compact tension (DCT) test and indirect tensile strength (IDT) test. The stress and strain relationship was assessed by the dynamic modulus test at different temperatures and frequencies. The extracted asphalt binder was evaluated by the dynamic shear rheometer (DSR). Pavement distress was predicted by pavement M-E analysis.

\section{Materials and Methods}

\subsection{Materials and Mixture Design}

The aggregate used in this study was obtained from Houghton, MI, USA. The three types of aggregate-minus screen, natural sand, and washed natural sand-were blended to produce the design gradation. Figure 1 displayed the detailed aggregate gradation of the flexible pavement. The asphalt PG 58-34 was used in this study, and its viscosity at $135^{\circ} \mathrm{C}$ is $0.398 \mathrm{~Pa} \cdot \mathrm{S}, \mathrm{G}^{*} / \sin (\delta)$ of unaged asphalt at $58^{\circ} \mathrm{C}$ is $1.542 \mathrm{kPa}, \mathrm{G}^{*} / \sin (\delta)$ of RTFO aged binder at $58{ }^{\circ} \mathrm{C}$ is $4.15 \mathrm{kPa}$, and $\mathrm{G}^{*} / \sin (\delta)$ of PAV aged binder at $16^{\circ} \mathrm{C}$ is $2.354 \mathrm{kPa}$. All the basic properties of the asphalt binder satisfied the specification requirement. The optimum asphalt binder content is $5.75 \%$. The scrap rubber and nylon fiber modified asphalt mixture was compacted with $7 \%$ air void by the Superpave gyrator compactor and compared with the conventional asphalt mixture.

The scrap rubber and nylon fiber used in the project are shown in Figure 2. The surface of the crumb rubber was treated to enhance the rubber-incorporated asphalt's workability. Nylon fiber from recycled tires was used in this project. 


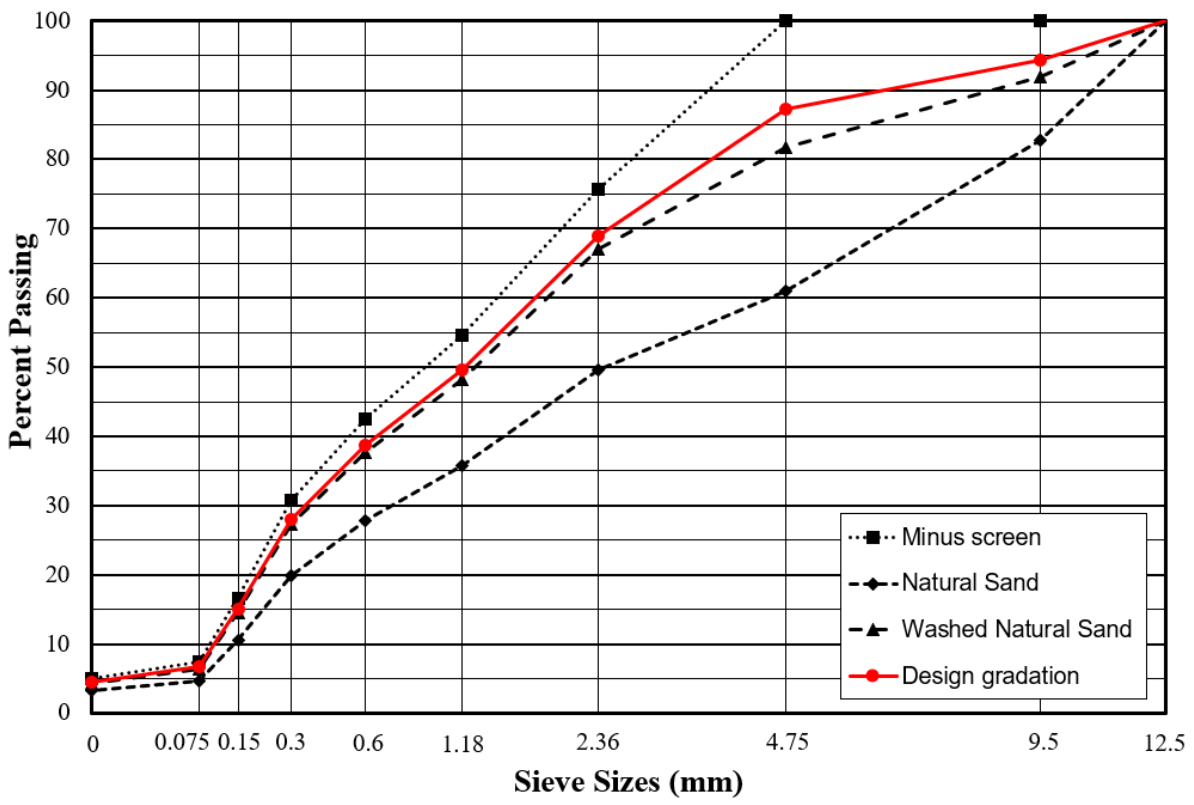

Figure 1. Aggregate gradation of the selected asphalt mixtures.

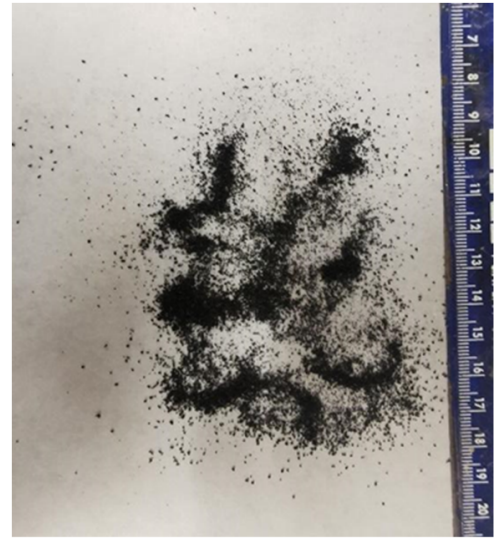

(a)

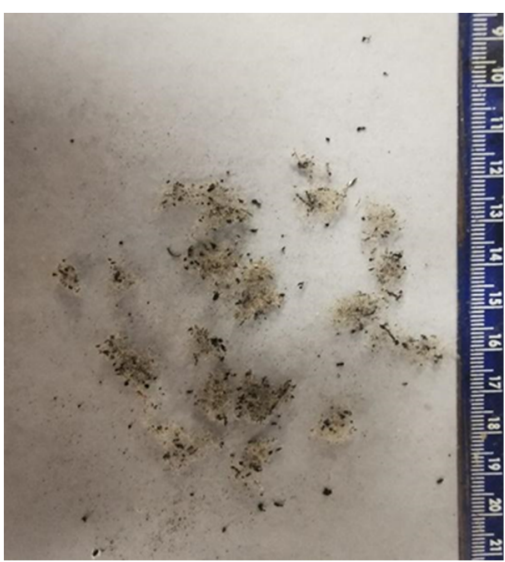

(b)

Figure 2. Scrap tire rubber and nylon fiber that were used in this study: (a) scrap tire rubber, (b) nylon fiber.

\subsection{Scrap Rubber and Nylon Fiber Modified WMA Preparation}

To help the nylon fiber distribute uniformly in asphalt, the nylon fiber and the Sasobit additive were incorporated into the PG 58-34 asphalt and mixed with the high-speed mixer at $170{ }^{\circ} \mathrm{C}$ for one hour. Then, the nylon fiber modified warm asphalt was mixed with aggregate and scrap tire rubber (10 wt.\% of asphalt binder) to produce scrap tire rubber and nylon fiber modified warm asphalt mixture. The Superpave gyratory compactor compacted the scrap tire rubber and nylon fiber modified warm asphalt mixture to the air void of $7 \%$. The details of the preparation process are shown in Figure 3. The conventional asphalt binder also was mixed in the high-speed mixer at $170{ }^{\circ} \mathrm{C}$ for one hour to condition it for comparison with the nylon fiber modified warm asphalt. 


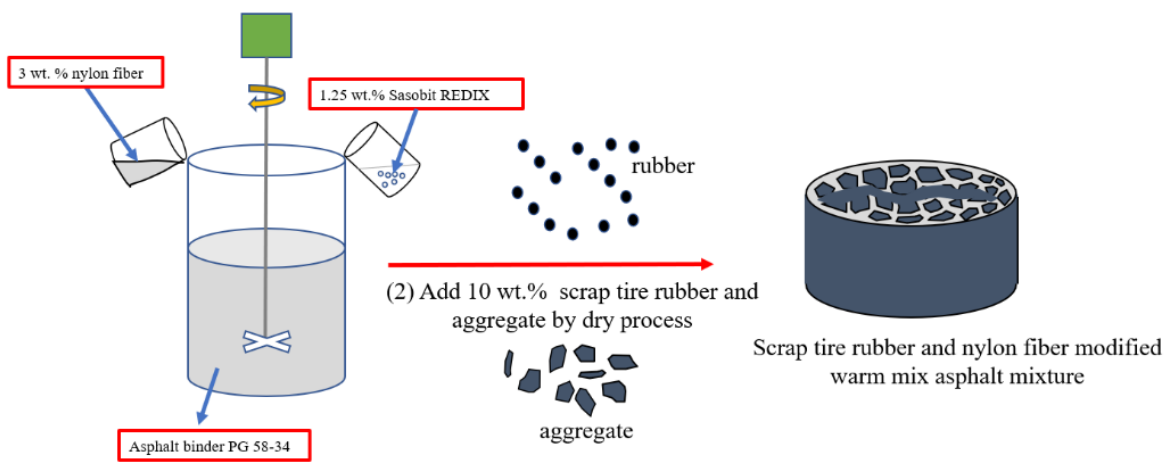

(1) Add nylon fiber, Sasobit REDIX

Figure 3. Preparation of scrap tire rubber and nylon fiber modified WMA.

\subsection{Traffic Inputs and Local Calibration Factors}

Pavement M-E analysis can be used to forecast pavement performance over time [17]. AASHTOWare Pavement Mechanistic-Empirical (ME) Design software has a considerable impact on asphalt pavement distress prediction. Average annual daily truck traffic (AADTT) is a critical input parameter when using Pavement M-E Design for flexible pavement design. Two different traffic inputs were implied to reflect the low (AADTT: 1000) and medium (AADTT: 2000) traffic levels in Michigan. Design life for the standard lane was approximated at 20 years. The number of lanes for the pavement was 2 , and the operational speed limit was $65 \mathrm{mph}(105 \mathrm{~km} / \mathrm{h})$. The percentage of trucks traveling in the direction for medium and heavy traffic level was $51 \%$. The percentage of trucks in the design lane was $92 \%$ with medium traffic level, and $65 \%$ with heavy traffic level. The traffic growth rate was $2 \%$ compound. Data from Buth et al. [18] were used to determine the distribution of the vehicle class, monthly and hourly adjustment factors, and distributions of the axle load.

\subsection{Research Methodology}

The laboratory mixture experiments can be summarized as follows: the dynamic modulus $\left(\left|\mathrm{E}^{*}\right|\right)$ test, the indirect tensile strength test, the disk-shape compact tension test, and the asphalt binder's dynamic shear modulus $\left(\left|\mathrm{G}^{*}\right|\right)$ derived from the loose material. Pavement M-E analysis was then proposed as a method for predicting pavement distresses. The technical flowchart is displayed in Figure 4, and the details are described as follows.

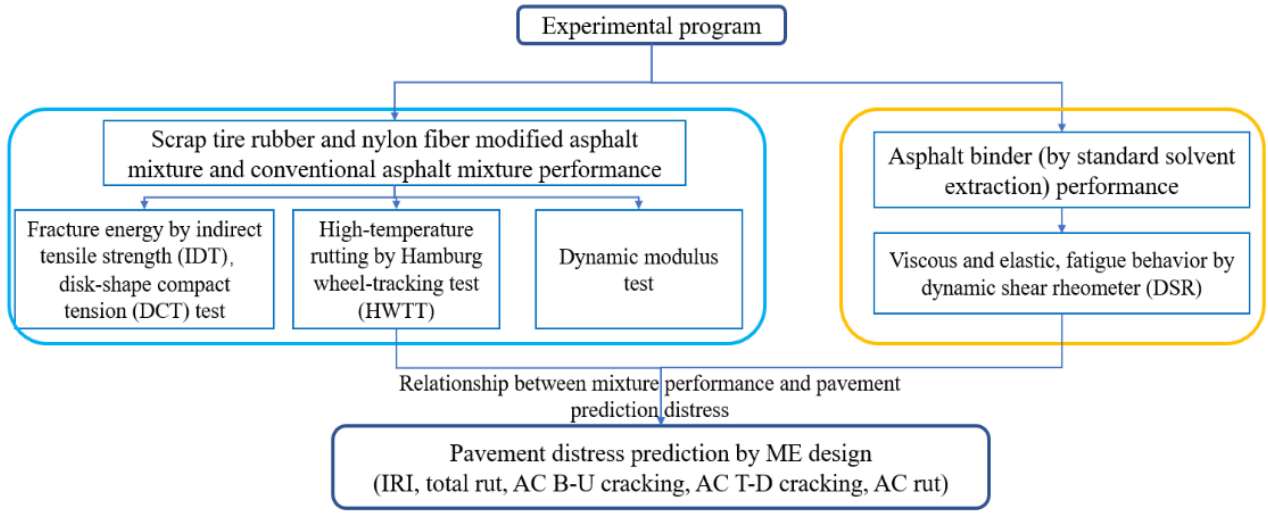

Figure 4. The technical flowchart of this study. 


\subsection{Test Program}

\subsubsection{Dynamic Modulus}

Scrap rubber and nylon fiber modified asphalt mixture is a viscoelastic composite material composed of stone, sand, viscous asphalt binder, and additives (scrap rubber and nylon fiber). Dynamic modulus is the main material property used in the program for Pavement ME Design. The loose mixture was compacted at least three replicates to $7 \pm 0.5 \%$ air void content with a $150 \mathrm{~mm}$ diameter and a $170 \mathrm{~mm}$ height, and the samples were cored with a $100 \mathrm{~mm}$ diameter and a $150 \mathrm{~mm}$ height and obtained air void content. The tests were conducted at temperatures of $-10,4,21,37$, and $54^{\circ} \mathrm{C}$ and at loading frequencies of 0.1 , $0.5,1.0,5,10$, and $25 \mathrm{~Hz}$ at each temperature. The dynamic modulus ( $\left.\left|\mathrm{E}^{*}\right|\right)$ master curve was obtained based on the dynamic modulus results. This test is according to AASHTO T 342 [19].

\subsubsection{Disk-Shaped Compact Tension (DCT) Test}

Fracture energy can be utilized to indicate the asphalt mixture's low-temperature cracking properties. The DCT test was utilized to estimate the fracture energy of scrap rubber and nylon fiber modified WMA and conventional asphalt mixture. The area under the displacement vs. load curve from the beginning to the end was obtained to calculate the fracture energy. This test follows ASTM standard D7313-20 [20]. The asphalt mixture was compacted to $7 \%$ air void with a $50 \mathrm{~mm}$ height and $150 \mathrm{~mm}$ diameter. Three replicate samples were prepared for the DCT test.

\subsubsection{Hamburg Wheel-Track Testing (HWTT) Test}

Permanent deformation of asphalt pavement is a vital signal of distress to indicate pavement qualities. The HWTT test was applied to evaluate the rutting performance of scrap rubber and nylon fiber modified WMA and convention asphalt mixture under a repeated wheel load. The results were used to reveal the rutting, moisture susceptibility, and stripping performance of the asphalt mixture. The test was performed according to AASHTO T 324 [21]. The sample had a height of $60 \mathrm{~mm}$ and a width of $150 \mathrm{~mm}$. The mixture was compressed to $7 \%$ air void, and three replicate samples for the HWTT test were generated.

\subsubsection{Indirect Tensile Strength (IDT)}

Indirect tensile strength can be used to reflect the tensile resistance of the field condition based on the field core. This test follows the standard AASHTO T 322 [22]. The samples were conditioned at $-10^{\circ} \mathrm{C}$ for $2 \mathrm{~h}$, and the IDT test was conducted. The loading speed was $12.5 \mathrm{~mm} / \mathrm{min}$. At least three field cores were used for the test results. The failure energy was calculated based on the load and displacement curve. Equation (1) of failure energy of asphalt mixture is shown below:

$$
\mathrm{G}_{\mathrm{f}}=\frac{\mathrm{W}_{\mathrm{f}}}{\mathrm{D} \times \mathrm{t}} \times 10^{6}
$$

where $G_{f}$ is failure energy (Joules $/ \mathrm{m}^{2}$ ), $W_{f}$ is work of failure (Joules), D is specimen diameter $(\mathrm{mm})$, and $\mathrm{t}$ is specimen thickness $(\mathrm{mm})$.

\subsubsection{Dynamic Shear Rheometer (DSR)}

The dynamic shear rheometer was used to determine the viscosity and elastic properties of asphalt at medium and high temperatures [23]. The tests were conducted at various temperatures and loading frequencies [24]. All of the unaged asphalt was placed in cylindrical glass bottles and placed in an oven on a rotating carriage. The rolling thin-film oven (RTFO) process matured the samples for $85 \mathrm{~min}$ as the carriage moved within the oven at $325^{\circ} \mathrm{F}\left(163^{\circ} \mathrm{C}\right)$ [25]. The long-term aged asphalt binder was stimulated by the pressure aging vessel (PAV) [26]. The results of the laboratory test results were used as M-E 
inputs in the Pavement ME Design software. The test findings were based on at least three samples, and the complex shear modulus $\left(\left|\mathrm{G}^{*}\right|\right)$ master curve was obtained based on the DSR results.

\subsubsection{Pavement M-E Distress Prediction}

To assess the differences in asphalt binder performance between rubber-nylon fiber modified asphalt pavement and conventional asphalt pavement, Pavement ME Design was utilized. According to the Pavement ME Design user guide's recommendation, the climate was applied at the close place. The specific calibration factor was calculated according to the "MDOT User Guide for Mechanistic-Empirical Pavement Design 2021" [27]. Table 1 shows the details of the pavement design information employed on this road. The creep compliance used in Pavement ME Design is according to the transfer process by S.W. Park and R.A. Shapery [28].

Table 1. Pavement structure and thickness used in this study.

\begin{tabular}{cc}
\hline Layer Types and Thickness & Pavement Structure \\
\hline Surface layer $(3.8 \mathrm{~cm})$ & Conventional or rubber and nylon fiber \\
modified warm mix asphalt (WMA) \\
Leveling course $(5 \mathrm{~cm})$ & Dense mix asphalt mixture \\
Asphalt base course $(17.8 \mathrm{~cm})$ & Dense mix asphalt mixture \\
Aggregate base $(15.2 \mathrm{~cm})$ & Sandwich granular \\
Aggregate base $(25.4 \mathrm{~cm})$ & Crushed stone \\
Aggregate subbase $(15.2 \mathrm{~cm})$ & Crushed gravel \\
subgrade & Semi-infinite
\end{tabular}

Note: The surface layer Pavement ME Design inputs are based on the laboratory test results. All the left layer Pavement ME Design inputs are based on the recommended reference value from MDOT.

\section{Results and Discussions}

\subsection{Complex Shear Modulus ( $\left.\left|G^{*}\right|\right)$ of Binders}

The complex shear modulus $\left(\left|\mathrm{G}^{*}\right|\right)$ and phase angle master curve of base asphalt binder and extract asphalt binder from rubber and nylon fiber modified WMA are displayed in Figure 5. Figure 5a shows the results of the PAV aged asphalt binder's complex shear modulus $\left(\left|G^{*}\right|\right)$ and phase angle. It shows that the rubber and nylon fiber modified warm mix asphalt had a lower complex shear modulus and higher phase angle than base asphalt and that the stiffness of the base binder increased more than that of nylon fiber modified warm mix asphalt at medium temperature after long-term aging. The main reason behind this is that the base binder incorporated with rubber and nylon fiber increased the viscous component. This indicates that the rubber and nylon fiber modified warm mix asphalt has better fatigue performance under loading. Figure $5 b$ depicts the RTFO aged asphalt binder's complex shear modulus $\left(\left|G^{*}\right|\right)$ and phase angle results. The results reveal that rubber and nylon fiber modified warm mix asphalt had a lower phase angle and a larger complex shear modulus than base asphalt after the short-term aging process. This means the rubber and nylon fiber modified warm mix asphalt was stiffer than base asphalt at high temperatures. It indicates that the rubber and nylon fiber modified warm mix asphalt has rutting resistance superior to that of the base asphalt binder. The rubber and nylon fiber particle in the asphalt increases the deformation resistance under loading. 

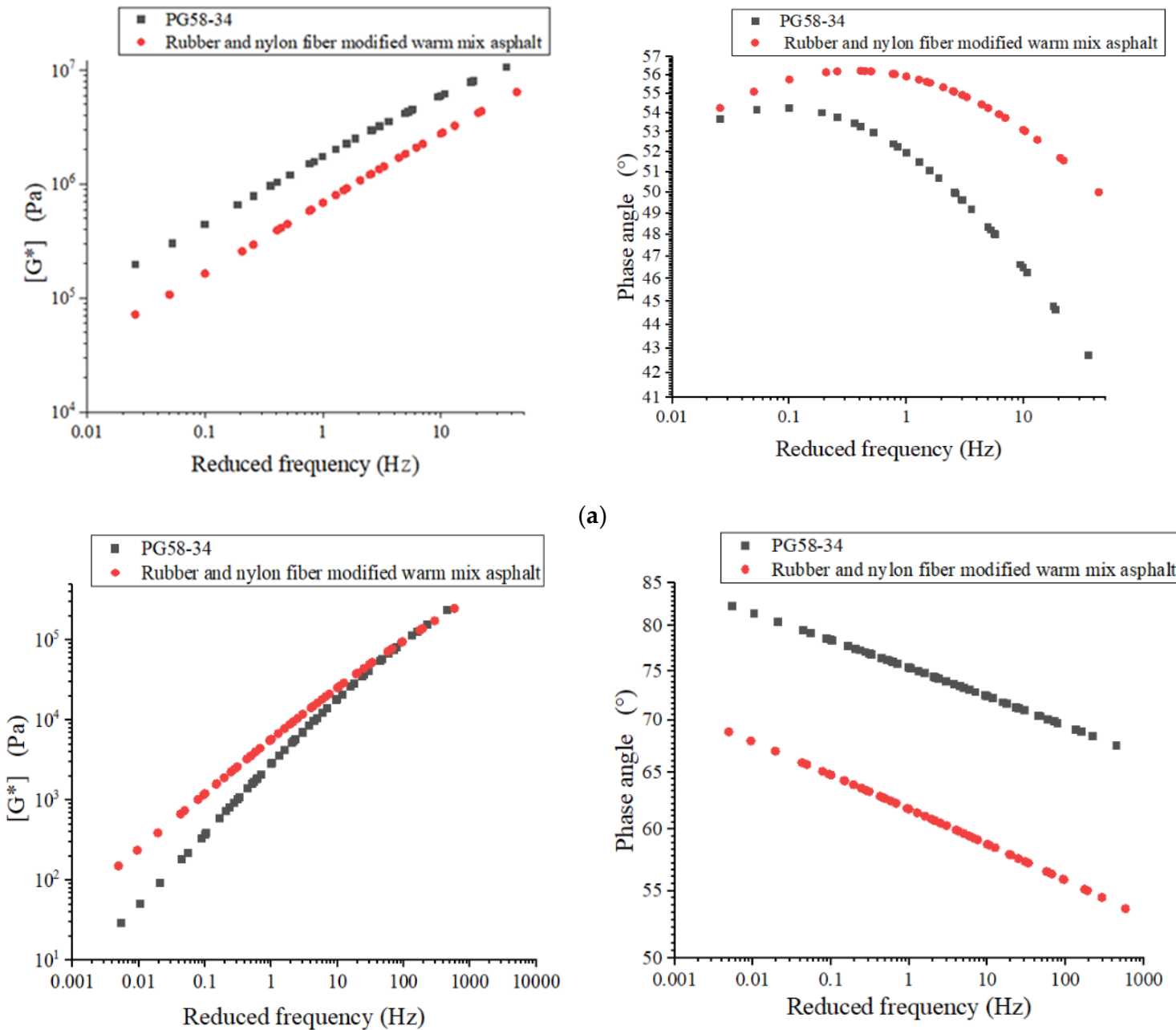

(a)

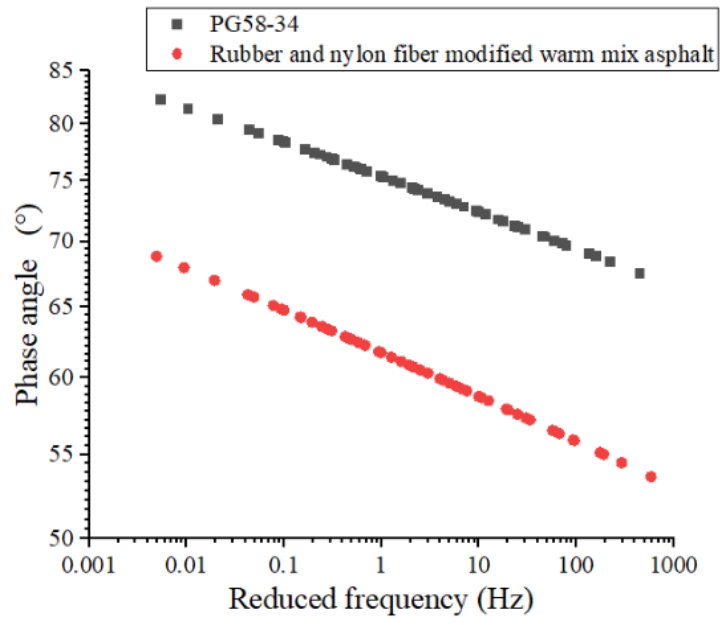

(b)

Figure 5. The master curve of different types of asphalt binder: (a) The complex shear modulus (left) and phase angle (right) master curve at $19{ }^{\circ} \mathrm{C}$ (PAV aged), (b) The complex shear modulus (left) and phase angle (right) master curve at $58{ }^{\circ} \mathrm{C}$ (RTFO aged).

\subsection{DCT Test Results}

The results of the disc-shaped compact tension test at low temperature are shown in Figure 6. The maximum load at the cracking point could be used to represent the cracking resistance of the asphalt mixture before cracking occurs. The DCT test load vs. displacement curve is shown in Figure 6a. It shows that the maximum load of rubber and nylon fiber modified WMA was $26 \%$ higher than that of the conventional asphalt mixture. The maximum CMOD could indicate the propagation in the asphalt mixture after the cracking occurs. It also shows that the maximum CMOD of rubber and nylon fiber modified WMA was $23.8 \%$ higher than that of the conventional asphalt mixture. The area under the load and displacement curve is the fracture energy. The cracking resistance of the asphalt mixture could be reflected as fracture energy. The fracture energy results of rubber and nylon fiber modified WMA and conventional HMA are shown in Figure 6b. It reveals that the fracture of rubber and nylon fiber modified WMA was $7.7 \%$ higher than that of the conventional asphalt mixture. This may be caused by the rubber and nylon fiber increasing the cracking resistance of the asphalt mixture. 


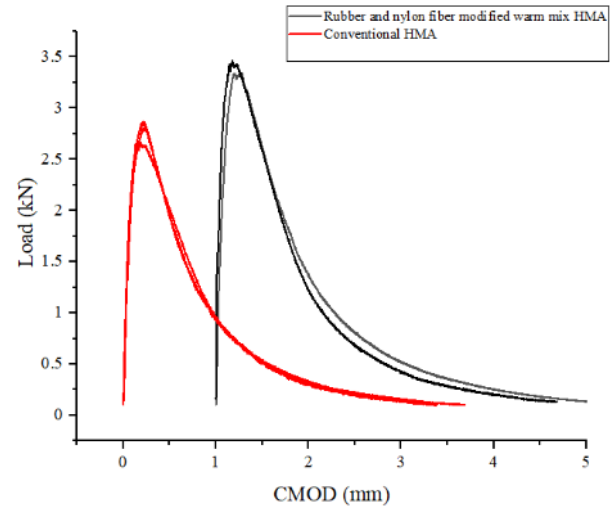

(a)

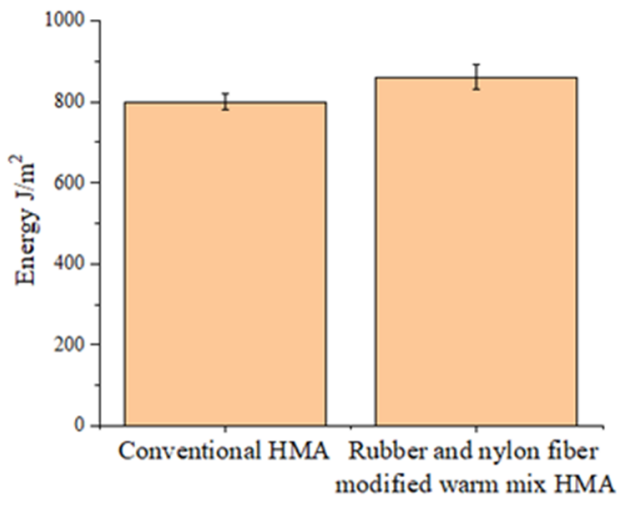

(b)

Figure 6. The DCT test results and fracture energy of various sorts of HMA: (a) DCT test results, (b) fracture energy results.

\subsection{HWDT Test Results}

The HWDT test results of rubber and nylon fiber modified WMA and conventional HMA are shown in Figure 7. The rubber and nylon fiber modified WMA had better rutting resistance than the conventional HMA. When the rutting depth reached up to $20 \mathrm{~mm}$, the number of wheel passes of rubber and nylon fiber modified WMA was 2.17 times higher than that of conventional HMA. Meanwhile, the stripping point of rubber and nylon fiber modified WMA was 5.8 times higher than that of conventional HMA. This indicates that the rubber and nylon fiber modified WMA had better moisture susceptibility performance than conventional HMA. The main reason behind this is that the rubber and nylon fiber in the asphalt mixture improves the stiffness and anti-stripping properties.

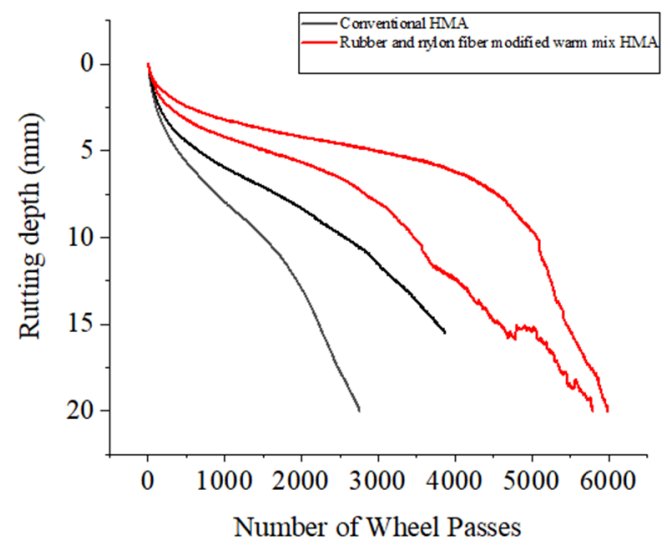

(a)

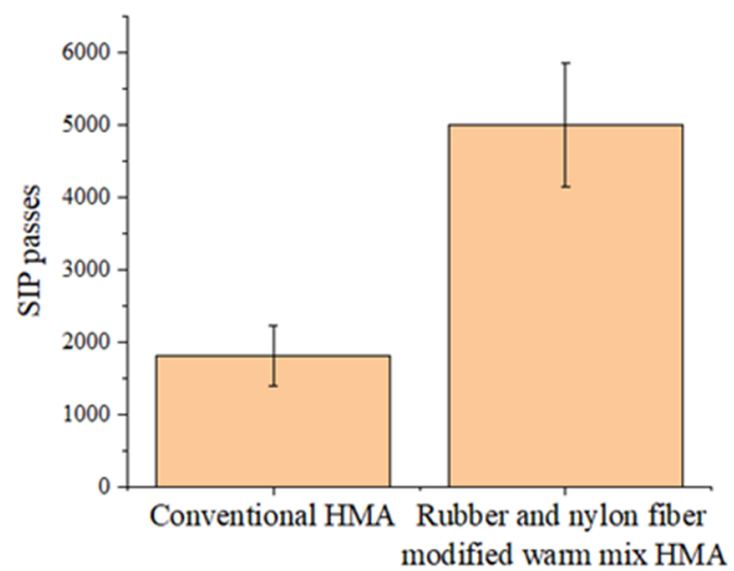

(b)

Figure 7. The HWDT test results of different types of asphalt mixture: (a) rutting depth vs. the number of wheel passes, (b) SIP passes results of various types of asphalt mixture.

\subsection{Dynamic Modulus Results}

The Master curve of dynamic modulus of nylon fiber modified WMA and conventional asphalt mixture are shown in Figure 8. The asphalt mixture's low-temperature performance is shown by the asphalt mixture's high reduced frequency. The asphalt mixture's hightemperature capability was demonstrated by its low reduced frequency; the high value at low reduced frequency means better rutting resistance. The dynamic modulus of conventional HMA was 21.8-103\% times lower than that of rubber and nylon fiber modified 
WMA. This indicates that the rubber and nylon fiber in the asphalt mixture improves the stiffness of rubber and nylon fiber modified WMA.

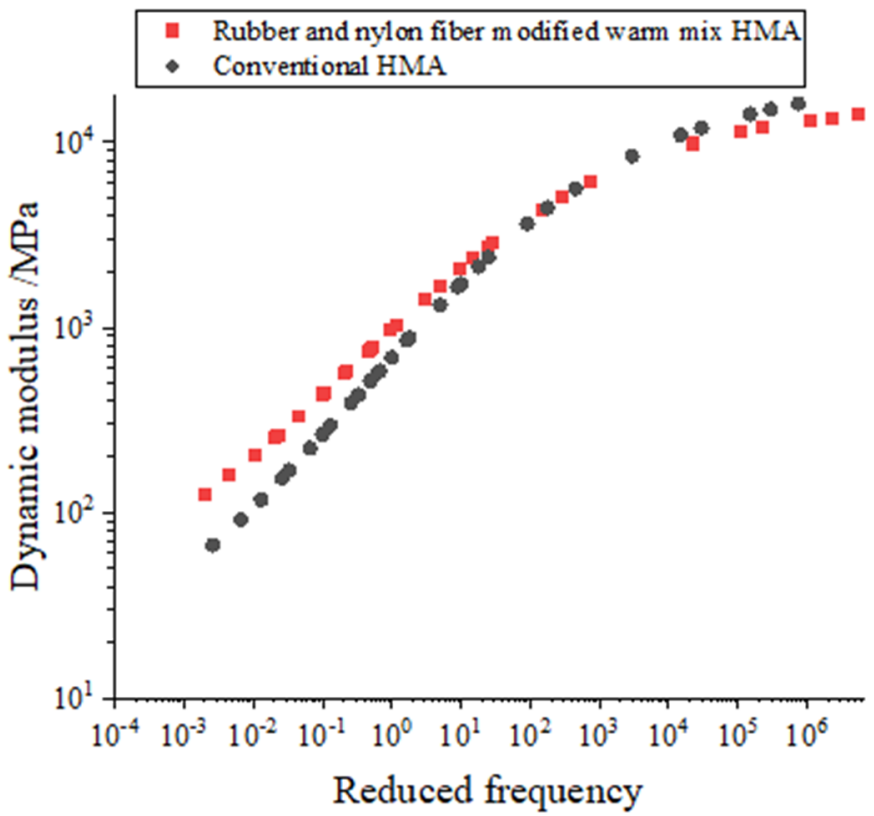

Figure 8. Dynamic modulus Master curve at $21^{\circ} \mathrm{C}$ for different types of asphalt mixture.

\subsection{IDT Strength and Failure Energy}

The indirect tensile strength results of rubber and nylon fiber modified WMA and conventional HMA are shown in Figure 9. The IDT strength of conventional HMA was $22.7 \%$ higher than that of rubber and nylon fiber modified WMA. In contrast, the failure energy of conventional HMA was $24.3 \%$ higher than that of rubber and nylon fiber modified WMA. The rubber particle decreases the stiffness of the asphalt mixture at low temperature, which causes the IDT strength to decrease, but the rubber and nylon particle increases the asphalt mixture's fracture resistance.

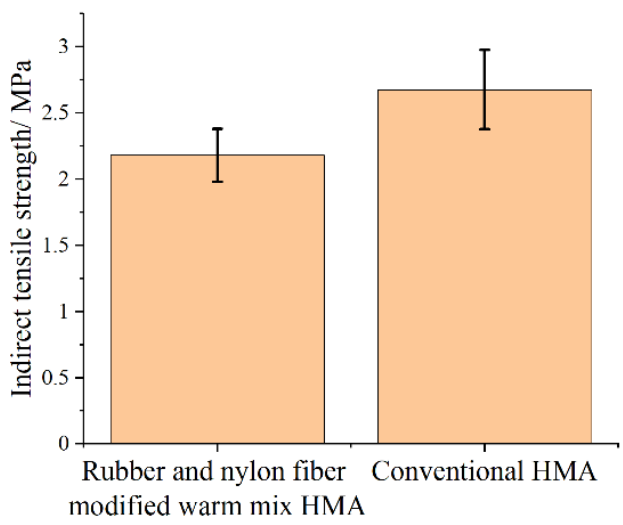

(a)

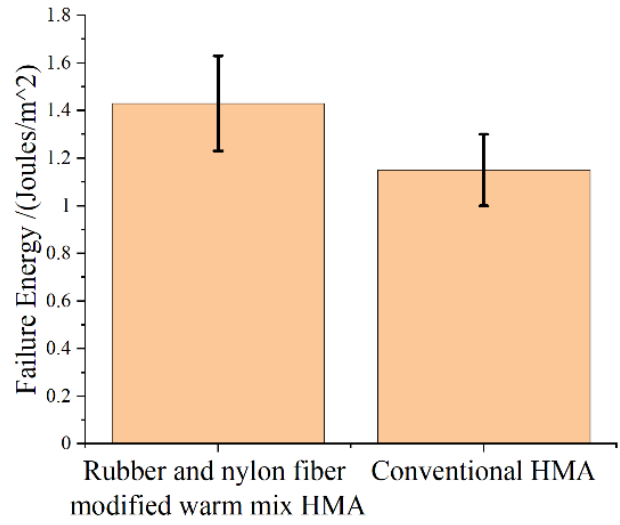

(b)

Figure 9. IDT strength and failure energy for various types of HMA: (a) IDT strength for different types of asphalt mixture, (b) failure energy for different types of asphalt mixture.

\subsection{Pavement Distress Prediction Results}

The pavement distress prediction results of rubber and nylon fiber modified WMA and conventional HMA are shown in Figure 10. The details of the M-E input for rubber and nylon fiber modified WMA and conventional HMA are shown in Tables 2 and 3. It could be concluded that if the AADTT traffic increased from 1000 to 2000, the distress for 
rubber and nylon fiber modified WMA and conventional HMA would increase, except for the AC thermal cracking. The IRI, total rutting, AC B-U cracking, AC thermal cracking, and $A C$ rutting distress of nylon fiber modified WMA increased $6.06 \mathrm{in} / \mathrm{mile}, 0.09 \mathrm{in}, 2.6 \%$ lane area, and 0.08 in., respectively. The IRI, total rutting, AC B-U cracking, AC thermal cracking, and AC rutting distress of conventional HMA increased $6.15 \mathrm{in} / \mathrm{mile}, 0.08 \mathrm{in}, 2$. $61 \%$ lane area, and 0.08 in., respectively. This means the rubber and nylon fiber modified WMA could help to improve the IRI and AC fatigue cracking performance with increased traffic volume. Moreover, the rubber and nylon fiber modified WMA helped to improve the IRI and AC cracking and rutting performance at the same traffic level compared with the conventional asphalt mixture. Taking AADTT equaling 1000 as an example, the IRI, AC $\mathrm{B}-\mathrm{U}$ cracking, AC thermal cracking, and AC rutting distress of the rubber and nylon fiber modified WMA decreased $0.38 \mathrm{in} / \mathrm{mile}, 0.04 \%$ lane area, $0.01 \%$ lane area, and $0.01 \mathrm{in} / \mathrm{mile}$, respectively. It should be mentioned that the extracted binder results used in Pavement ME Design could not wholly reflect the asphalt binder performance of the rubber and nylon fiber modified WMA. The main reason is that the left rubber particle or nylon fiber particle in the loose aggregate which does not have contact with the asphalt binder would not be considered in the asphalt mixture. Therefore, the M-E results do not fully reveal the prediction distress. In addition, the DSR data used in pavement M-E analysis are based on the extracted asphalt binder from the rubber and nylon fiber modified WMA. It may not fully reflect the properties of the rubber and nylon fiber modified asphalt because there are still some rubber particles left in the solvent.

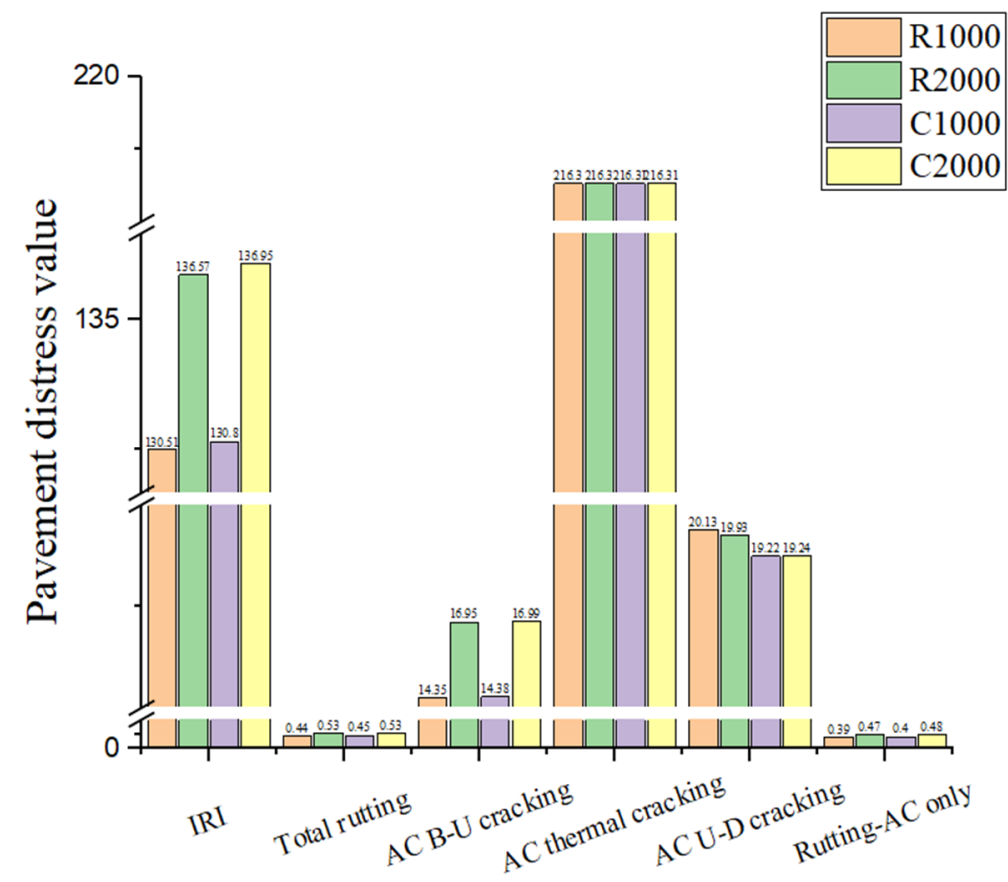

Figure 10. Pavement distress prediction results for different types of asphalt mixture. Note: R1000 means rubber and nylon fiber modified WMA at 1000 AADTT traffic levels. R2000 means rubber and nylon fiber modified WMA at 2000 AADTT traffic level. C1000 means conventional HMA at 1000 AADTT traffic level. C2000 means conventional HMA at 2000 AADTT traffic, IRI smoothness (in $/ \mathrm{mile}$ or $0.016 \mathrm{~m} / \mathrm{km}$ ), total rut (in or $0.0254 \mathrm{~m}$ ), AC B-U (bottom-up) cracking (\% lane area), and $\mathrm{AC}$ rut (in or $0.0254 \mathrm{~m}$ ). 
Table 2. Pavement ME Design inputs for rubber and nylon fiber modified WMA.

I E* | (MPa) Average Value Rubber and Nylon Fiber Modified WMA

\begin{tabular}{|c|c|c|c|c|c|c|c|}
\hline & F (Hz) & 0.1 & 0.5 & 1 & 5 & 10 & 25 \\
\hline \multicolumn{8}{|l|}{$\mathrm{T}\left({ }^{\circ} \mathrm{C}\right)$} \\
\hline-10 & & 6463.2 & 8848.3 & $10,010.1$ & $12,667.3$ & $14,253.0$ & $15,521.3$ \\
\hline 10 & & 2180.8 & 3261.2 & 3935.8 & 5773.4 & 6679.5 & 7673.6 \\
\hline 21 & & 511.5 & 718.1 & 947.1 & 1620.4 & 2061.0 & 2645.0 \\
\hline 37 & & 210.9 & 261.8 & 321.9 & 492.2 & 610.3 & 862.9 \\
\hline 54 & & 120.0 & 181.0 & 309.0 & 498.1 & 606.6 & 773.3 \\
\hline \multicolumn{4}{|c|}{ | G* I (MPa) Average Value } & \multicolumn{4}{|c|}{ Creep Compliance } \\
\hline $\begin{array}{c}\text { Temperature } \\
\left({ }^{\circ} \mathrm{C}\right)\end{array}$ & \multicolumn{2}{|c|}{ Binder $\mathrm{G}^{*}(\mathrm{~Pa})$} & $\begin{array}{c}\text { Phase angle } \\
\left({ }^{\circ}\right)\end{array}$ & \multirow{2}{*}{ Time (s) } & \multicolumn{3}{|c|}{ Temperature $\left({ }^{\circ} \mathrm{C}\right)$} \\
\hline 16 & & & 54.38 & & -20 & -10 & 0 \\
\hline 34 & & & 58.89 & 1 & $5.32 \times 10^{-7}$ & $1.04 \times 10^{-6}$ & $2.25 \times 10^{-6}$ \\
\hline 46 & & & 58.27 & 2 & $5.87 \times 10^{-7}$ & $1.21 \times 10^{-6}$ & $2.69 \times 10^{-6}$ \\
\hline 64 & & & 60.38 & 5 & $6.86 \times 10^{-7}$ & $1.46 \times 10^{-6}$ & $3.50 \times 10^{-6}$ \\
\hline 76 & & & 64.83 & 10 & $7.75 \times 10^{-7}$ & $1.74 \times 10^{-6}$ & $4.24 \times 10^{-6}$ \\
\hline \multirow{3}{*}{\multicolumn{4}{|c|}{$\left(-10^{\circ} \mathrm{C}\right)$ IDT strength: $2.676 \mathrm{MPa}$}} & 20 & $8.75 \times 10^{-7}$ & $2.07 \times 10^{-6}$ & $5.18 \times 10^{-6}$ \\
\hline & & & & 50 & $1.06 \times 10^{-6}$ & $2.59 \times 10^{-6}$ & $6.94 \times 10^{-6}$ \\
\hline & & & & 100 & $1.22 \times 10^{-6}$ & $3.15 \times 10^{-6}$ & $8.56 \times 10^{-6}$ \\
\hline
\end{tabular}

Table 3. Pavement ME Design inputs for conventional HMA.

\begin{tabular}{|c|c|c|c|c|c|c|}
\hline \multicolumn{7}{|c|}{ I E* I (MPa) Average Value Conventional HMA } \\
\hline & F (Hz) & 0.5 & 1 & 5 & 10 & 25 \\
\hline \multicolumn{7}{|l|}{$\mathrm{T}\left({ }^{\circ} \mathrm{C}\right)$} \\
\hline-10 & 6821.2 & 9806.8 & $11,183.8$ & $14,234.0$ & $15,584.8$ & $16,782.7$ \\
\hline 10 & 2548.0 & 4570.4 & 5806.5 & 8961.4 & $10,779.7$ & $12,600.4$ \\
\hline 21 & 296.5 & 512.7 & 600.3 & 1081.1 & 1476.7 & 2032.4 \\
\hline 37 & 142.6 & 182.0 & 219.4 & 444.7 & 532.5 & 755.6 \\
\hline 54 & 60.0 & 100.0 & 152.0 & 352.0 & 498.0 & 582.0 \\
\hline \multicolumn{3}{|c|}{ I G* I (MPa) Average Value } & \multicolumn{4}{|c|}{ Creep Compliance } \\
\hline $\begin{array}{c}\text { Temperature } \\
\left({ }^{\circ} \mathrm{C}\right)\end{array}$ & Binder $\mathrm{G}^{*}(\mathrm{~Pa})$ & $\begin{array}{c}\text { Phase angle } \\
\left({ }^{\circ}\right)\end{array}$ & \multirow{2}{*}{ Time (s) } & \multicolumn{3}{|c|}{ Temperature $\left({ }^{\circ} \mathrm{C}\right)$} \\
\hline 16 & $3,146,800$ & 48.63 & & -20 & -10 & 0 \\
\hline 34 & $78,249.67$ & 68.16 & 1 & $4.35 \times 10^{-7}$ & $7.94 \times 10^{-7}$ & $1.95 \times 10^{-6}$ \\
\hline 46 & $16,972.33$ & 71.25 & 2 & $4.64 \times 10^{-7}$ & $8.93 \times 10^{-7}$ & $2.35 \times 10^{-6}$ \\
\hline 64 & 2042.63 & 75.98 & 5 & $5.05 \times 10^{-7}$ & $1.05 \times 10^{-6}$ & $3.02 \times 10^{-6}$ \\
\hline 76 & 594.66 & 79.63 & 10 & $5.43 \times 10^{-7}$ & $1.21 \times 10^{-6}$ & $3.74 \times 10^{-6}$ \\
\hline \multirow{3}{*}{\multicolumn{3}{|c|}{$\left(-10^{\circ} \mathrm{C}\right)$ IDT strength: $2.18 \mathrm{MPa}$}} & 20 & $5.92 \times 10^{-7}$ & $1.41 \times 10^{-6}$ & $4.69 \times 10^{-6}$ \\
\hline & & & 50 & $6.65 \times 10^{-7}$ & $1.74 \times 10^{-6}$ & $6.32 \times 10^{-6}$ \\
\hline & & & 100 & $7.34 \times 10^{-7}$ & $2.09 \times 10^{-6}$ & $8.12 \times 10^{-6}$ \\
\hline
\end{tabular}




\section{Summary and Conclusions}

This study focused on the evaluation of the performance at both high and low temperatures of rubber and nylon fiber modified WMA based on the dynamic modulus test, Hamburg wheel-track testing test, disk-shaped compact tension test, indirect tensile strength test, and extracted asphalt binder rheology characteristics and predicted the pavement distress based on the mechanistic-empirical (M-E) pavement design method in Michigan's asphalt pavement. This study's findings can be summarized as follows:

(1) The test of dynamic modulus revealed that the rubber and nylon fiber modified WMA had higher stiffness compared with the conventional asphalt mixture at high temperature, which increases the rutting resistance at high temperatures. The dynamic modulus of conventional HMA was 21.8 103\% lower than that of rubber and nylon fiber modified WMA at high temperatures.

(2) The dynamic shear rheometer test results show that the rubber and nylon fiber modified warm mix asphalt has better rutting resistance and fatigue performance than the base asphalt binder. The rubber and nylon fiber particle in the asphalt increases the strength inside of the asphalt binder, which improves the deformation resistance and fatigue property under the load.

(3) The IDT and DCT test results show that the rubber and nylon fiber modified WMA had a higher crack resistance when compared with traditional asphalt mixtures. According to the findings of the DCT test, the failure energy of the rubber and nylon fiber modified WMA was $24.3 \%$ higher than that of the conventional HMA, and the fracture energy of the rubber and nylon fiber modified WMA was 7.7\% higher than the conventional asphalt mixture. This may be caused by the rubber and nylon fiber increasing the cracking resistance of the asphalt mixture.

(4) The HWDT test results show that the rubber and nylon fiber modified WMA has better rutting resistance and anti-striping than the conventional HMA. The number of wheel passes of rubber and nylon fiber modified WMA was 2.17 times higher than that of conventional HMA. Meanwhile, the stripping point of rubber and nylon fiber modified WMA was 5.8 times higher than that of conventional HMA. The main reason behind this is that the rubber and nylon fiber in the asphalt mixture improves the stiffness and anti-stripping properties.

(5) The pavement distress prediction results show that the rubber and nylon fiber modified WMA helps to improve the IRI and AC cracking and rutting performance at the same or increased traffic level compared with the conventional asphalt mixture. The results from the pavement M-E analysis show the same trend compared with the laboratory testing performance.

In summary, the implementation of the rubber and nylon fiber modified WMA increased the performance of asphalt pavement in terms of high-temperature rutting, cracking resistance at low-temperature, and fatigue properties. Moreover, the pavement M-E analysis showed a better prediction distress performance in comparison with the conventional HMA, so rubber and nylon fiber modified warm mix asphalt pavement could have better performance on low traffic volume roads compared with conventional asphalt pavement.

Author Contributions: Conceptualization, D.J., D.G., and Z.Y.; data curation, D.J., X.Z., and D.G.; methodology, D.J., D.G., and X.Z.; resources, Z.Y.; supervision, Z.Y.; writing-original draft, D.J.; writing-review and editing, D.J., D.G., X.Z., and Z.Y. All authors have read and agreed to the published version of the manuscript.

Funding: This work is sponsored by the Michigan Department of Environment, Great Lakes, and Energy (EGLE), Grant Contract Number: CU-1731057.

Institutional Review Board Statement: Not applicable.

Informed Consent Statement: Not applicable. 
Data Availability Statement: On reasonable request, the corresponding author will make the datasets generated during the current investigation available.

Acknowledgments: All the loose aggregate was obtained from Houghton County and the asphalt binder was obtained from Dickinson County.

Conflicts of Interest: The authors declare no conflict of interest.

\section{References}

1. Li, X.; Zhou, Z.; Ye, J.; Zhang, X.; Wang, S.; Diab, A. High-temperature creep and low-temperature relaxation of recycled asphalt mixtures: Evaluation and balanced mix design. Constr. Build. Mater. 2021, 310, 125222. [CrossRef]

2. Chen, J.; Dan, H.; Ding, Y.; Gao, Y.; Guo, M.; Guo, S.; Han, B.; Hong, B.; Hou, Y.; Hu, C.; et al. New innovations in pavement materials and engineering: A review on pavement engineering research 2021. J. Traffic Transp. Eng. (Engl. Ed.) 2021, 8, 815-999. [CrossRef]

3. Marconi, M.; Landi, D.; Meo, I.; Germani, M. Reuse of tires textile fibers in plastic compounds: Is this scenario environmentally sustainable? Procedia Cirp 2018, 69, 944-949. [CrossRef]

4. Ge, D.; Zhou, X.; Chen, S.; Jin, D.; You, Z. Laboratory Evaluation of the Residue of Rubber-Modified Emulsified Asphalt. Sustainability 2020, 12, 8383. [CrossRef]

5. Ogunbiyi, A.W.; Openibo, A.O.; Ojowuro, O.M. Smelting of Waste Nylon and Low-density Plastics (ldps) in a Fluidized Bed System. Procedia Environ. Sci. 2016, 35, 491-497. [CrossRef]

6. Wang, W.; Meng, L.; Leng, K.; Huang, Y. Hydrolysis of waste monomer casting nylon catalyzed by solid acids. Polym. Degrad. Stab. 2017, 136, 112-120. [CrossRef]

7. Yin, J.; Wu, W. Utilization of waste nylon wire in stone matrix asphalt mixtures. Waste Manag. 2018, 78, 948-954. [CrossRef] [PubMed]

8. Lee, S.J.; Rust, J.P.; Hamouda, H.; Kim, Y.R.; Borden, R.H. Fatigue Cracking Resistance of Fiber-Reinforced Asphalt Concrete. Text. Res. J. 2005, 75, 123-128. [CrossRef]

9. Taherkhani, H. Investigating the effects of nanoclay and nylon fibers on the mechanical properties of asphalt concrete. Civ. Eng. Infrastruct. J. 2016, 49, 235-249.

10. Yang, X.; You, Z.; Hasan, M.R.M.; Diab, A.; Shao, H.; Chen, S.; Ge, D. Environmental and mechanical performance of crumb rubber modified warm mix asphalt using Evotherm. J. Clean. Prod. 2017, 159, 346-358. [CrossRef]

11. Oliveira, J.R.; Silva, H.M.; Abreu, L.P.; Fernandes, S.R. Use of a warm mix asphalt additive to reduce the production temperatures and to improve the performance of asphalt rubber mixtures. J. Clean. Prod. 2013, 41, 15-22. [CrossRef]

12. Dinis-Almeida, M. and M.L. Afonso, Warm mix recycled asphalt-a sustainable solution. J. Clean. Prod. 2015, 107, 310-316. [CrossRef]

13. Airey, G.D.; Rahman, M.M.; Collop, A.C. Absorption of Bitumen into Crumb Rubber Using the Basket Drainage Method. Int. J. Pavement Eng. 2003, 4, 105-119. [CrossRef]

14. Rahman, M.M.; Airey, G.D.; Collop, A.C. Moisture Susceptibility of High and Low Compaction Dry Process Crumb RubberModified Asphalt Mixtures. Transp. Res. Rec. J. Transp. Res. Board 2010, 2180, 121-129. [CrossRef]

15. Chen, S.; Ge, D.; Jin, D.; Zhou, X.; Liu, C.; Lv, S.; You, Z. Investigation of hot mixture asphalt with high ground tire rubber content. J. Clean. Prod. 2020, 277, 124037. [CrossRef]

16. Xie, Z.; Shen, J. Fatigue Performance of Rubberized Stone Matrix Asphalt by a Simplified Viscoelastic Continuum Damage Model. J. Mater. Civ. Eng. 2016, 28, 04015162. [CrossRef]

17. Jin, D.; Ge, D.; Chen, S.; Che, T.; Liu, H.; Malburg, L.; You, Z. Cold In-Place Recycling Asphalt Mixtures: Laboratory Performance and Preliminary M-E Design Analysis. Materials 2021, 14, 2036. [CrossRef] [PubMed]

18. Buch, N.; Haider, S.W.; Brown, J.; Chatti, K. Characterization of Truck Traffic in Michigan for the New Mechanistic Empirical Pavement Design Guide; Michigan Department of Transportation, Construction and Technology Division: Lansing, MI, USA, 2009.

19. AASHTO T 342; Standard Method of Test for Determining Dynamic Modulus of Hot Mix Asphalt (HMA). American Association of State Highway Transportation Officials: Washington, DC, USA, 2011.

20. ASTM D7313-20; Standard Test Method for Determining Fracture Energy of Asphalt Mixtures Using the Disk-Shaped Compact Tension Geometry. American Society for Testing and Materials International: West Conshohocken, PA, USA, 2021.

21. AASHTO T 324; Standard Method of Test for Hamburg Wheel-Track Testing of Compacted Asphalt Mixtures. American Association of State Highway Transportation Officials: Washington, DC, USA, 2019.

22. AASHTO T 322; Standard Method of Test for Determining the Creep Compliance and Strength of Hot Mix Asphalt (HMA) Using the Indirect Tensile Test Device. American Association of State Highway Transportation Officials: Washington, DC, USA, 2007.

23. Jin, D.; Wang, J.; You, L.; Ge, D.; Liu, C.; Liu, H.; You, Z. Waste cathode-ray-tube glass powder modified asphalt materials: Preparation and characterization. J. Clean. Prod. 2021, 314, 127949. [CrossRef]

24. AASHTO T 315; Standard Method of Test for Determining the Rheological Properties of Asphalt Binder Using a Dynamic Shear Rheometer. American Association of State Highway Transportation Officials: Washington, DC, USA, 2020.

25. AASHTO T 240; Standard Method of Test for Effect of Heat and Air on a Moving Film of Asphalt Binder (Rolling Thin-Film Oven Test). American Association of State Highway Transportation Officials: Washington, DC, USA, 2009. 
26. AASHTO R 28; Standard Practice for Accelerated Aging of Asphalt Binder Using a Pressurized Aging Vessel (PAV). American Association of State Highway Transportation Officials: Washington, DC, USA, 2009.

27. MDOT. Michigan DOT User Guide for Mechanistic-Empirical Pavement Design; Michigan Department of Transportation: Lansing, MI, USA, 2021.

28. Park, S.; Schapery, R. Methods of interconversion between linear viscoelastic material functions. Part I-a numerical method based on Prony series. Int. J. Solids Struct. 1999, 36, 1653-1675. [CrossRef] 